\title{
Optimized Grad -Shafranov Reconstruction of a Magnetic Cloud Using STEREO-Wind Observations
}

\author{
C. Möstl · C.J. Farrugia · H.K. Biernat · M. Leitner • \\ E.K.J. Kilpua $\cdot$ A.B. Galvin · J.G. Luhmann
}

Received: 26 November 2008 / Accepted: 27 March 2009 / Published online: 23 April 2009

(C) The Author(s) 2009. This article is published with open access at Springerlink.com

\begin{abstract}
We present results on the geometry of a magnetic cloud (MC) on 23 May 2007 from a comprehensive analysis of Wind and STEREO observations. We first apply a GradShafranov reconstruction to the STEREO-A plasma and magnetic field data, delivered by the PLASTIC and IMPACT instruments. We then optimize the resulting field map with the aid of observations by Wind, which were made at the very outer boundary of the cloud, at a spacecraft angular separation of $6^{\circ}$. For the correct choice of reconstruction parameters such as axis orientation, interval and grid size, we find both a very good match between the predicted magnetic field at the position of Wind and the actual observations as well as consistent timing. We argue that the reconstruction captures almost the full extent of the cross-section of the cloud. The resulting shape transverse to the invariant axis consists of distorted ellipses and is slightly flattened in the direction of motion. The MC axis is inclined at $-58^{\circ}$ to the ecliptic with an axial field strength of $12 \mathrm{nT}$. We derive integrated axial fluxes and currents with increased precision, which we contrast with the results from linear force-
\end{abstract}

STEREO Science Results at Solar Minimum

Guest Editors: Eric R. Christian, Michael L. Kaiser, Therese A. Kucera, O.C. St. Cyr.

C. Möstl $(\bowtie) \cdot$ H.K. Biernat

Space Research Institute, Austrian Academy of Sciences, Graz 8042, Austria

e-mail: christian.moestl@oeaw.ac.at

C. Möstl · H.K. Biernat

Institute of Physics, University of Graz, Graz 8010, Austria

C.J. Farrugia · A.B. Galvin

Space Science Center and Dept. of Physics, University of New Hampshire, Durham, NH 03824, USA

M. Leitner

Institute for Astro- and Particle Physics, University of Innsbruck, 6020 Innsbruck, Austria

E.K.J. Kilpua

Department of Physics, University of Helsinki, 00014 Helsinki, Finland

J.G. Luhmann

Space Science Laboratory, University of California, Berkeley, CA 94720, USA 
free fitting. The helical geometry of the MC with almost constant twist $\left(\approx 1.5\right.$ turns $\left.\mathrm{AU}^{-1}\right)$ is not consistent with the linear force-free Lundquist model. We also find that the cloud is non-force-free $\left(\left|\boldsymbol{J}_{\perp}\right| /\left|\boldsymbol{J}_{\|}\right|>0.3\right)$ in about a quarter of the cloud cross sectional area, particularly in the back part which is interacting with the trailing high speed stream. Based on the optimized reconstruction we put forward preliminary guidelines for the improved use of single-spacecraft Grad-Shafranov reconstruction. The results also give us the opportunity to compare the CME direction inferred from STEREO/SECCHI observations by Mierla et al. (Solar Phys. 252, 385, 2008) with the three-dimensional configuration of the MC at 1 AU. This yields an almost radial CME propagation from the Sun to the Earth.

\section{Introduction}

Interplanetary coronal mass ejections (ICMEs) are the in situ-observed counterparts of coronal mass ejections (CMEs) as imaged by solar coronagraphs. Magnetic clouds are a subset of ICMEs characterized by a smooth rotation of the magnetic field vector with a stronger-thanaverage total field, low proton temperatures, and a low ratio of the plasma-to-magnetic pressure (Burlaga et al., 1981). It has been suggested that magnetic clouds may form the central flux ropes of ICMEs, with the classification of the observed signatures depending on the trajectory of the spacecraft (Cane and Richardson, 2003; Jian et al., 2006; Gopalswamy, 2006; Reinard, 2008). However, it seems unlikely that every ICME has an MC core (Riley et al., 2006). Aside from this, many other unsolved questions regarding the three-dimensional shape of MCs in the heliosphere remain. It has been shown from a variety of independent studies that MCs are magnetic flux ropes of locally straight cylindrical geometry (Burlaga, 1988; Farrugia, Osherovich, and Burlaga, 1995; Shodhan et al., 2000; Liu et al., 2008). On the large-scale an MC is thought to form a bent flux rope extending from the Sun into interplanetary space (Burlaga, Lepping, and Jones, 1990; Marubashi, 1997; Bothmer and Schwenn, 1998) with its feet possibly still connected to the Sun (e.g., Farrugia et al., 1993b, and references therein). Also the possibility that the forward regions of MCs are peeled off by reconnection with the interplanetary magnetic field during their propagation from Sun to Earth has been recently put forward (Dasso et al., 2006, 2007; Gosling et al., 2007; Möstl et al., 2008). Previously, Farrugia et al. (2001) had studied a reconnection layer at the leading edge of a magnetic cloud separating the cloud from the ejected material ahead of it. This possibility should be borne in mind as it influences the MC magnetic flux budget, which is important for establishing the Sun - Earth link (e.g. Longcope et al., 2007; Qiu et al., 2007; Démoulin, 2008; Möstl et al., 2009). However, aside from very few events (Mulligan et al., 1999; Mulligan and Russell, 2001), the shape of the MC cross-section and its longitudinal extent are not well known due to a lack of suitable multi-spacecraft observations.

In addition to their unique stereoscopic imaging capabilities, the two Solar TErrestrial Relations Observatory (STEREO) spacecraft drifting away from Earth in the ecliptic at the rate of $22^{\circ} \mathrm{yr}^{-1}$ also provide unprecedented two-point in situ measurements of the local interplanetary magnetic field, the solar wind plasma flow parameters and composition. Mission phase 1, from January 2007, when the STEREO separation was $0.05^{\circ}$, to April 2008 (separation $50^{\circ}$ ), is the most promising for observing MCs at more than one spacecraft (Kaiser et al., 2008). Multi-spacecraft analyses have been conducted for two magnetic cloud events, those on 22 May 2007 (Liu et al., 2008; Kilpua et al., 2009; Möstl et al., 2009) and on 23 May 2007 (Kilpua et al., 2009). Both MCs were strongly inclined $\left(\approx 50^{\circ}\right.$ and $\approx-60^{\circ}$, respectively) to the ecliptic and thus the STEREO spacecraft, separated by $9^{\circ}$, crossed the MC approximately perpendicular to its axis. These efforts have 
shown that the cross-section of these two MCs is indeed "flattened", i.e. elongated in the plane transverse to the $\mathrm{MC}$ axis and its direction of motion, but to a lesser extent than what has previously been thought (Riley and Crooker, 2004; Liu et al., 2006). This discrepancy might be caused by their highly inclined axes as well as their interaction with high-speed solar wind streams during solar minimum.

It is the aim of this paper to demonstrate quantitatively the ability of the method of Grad-Shafranov (GS) reconstruction (Hau and Sonnerup, 1999; Hu and Sonnerup, 2002; Sonnerup et al., 2006) to retrieve an approximately correct cross-section of a magnetic cloud. To this end, we revisit the magnetic cloud event on 23 May 2007 and add new elements to previous analysis. Basic GS-reconstruction of the 23 May 2007 event has already been discussed by Kilpua et al. (2009). Here, we extend this analysis to demonstrate further that this MC is non-force free and its twisted field lines have an unexpected property, and to provide a set of guidelines for future use of the GS method. It has also been claimed that the GS method has a limited spatial domain which does not allow the full cross-section to be recovered (Riley et al., 2004). Contrary to this, we will show that for this average-sized magnetic cloud (scale size in the radial direction $=0.12 \mathrm{AU}$ ) the reconstruction from STEREO-A data, constrained by Wind observations, covers almost the complete cloud cross-section. We also consider that GS is a static method which does not include the often reported expansion of the MCs (e.g. Farrugia et al., 1992, 1993a, 1997; Dasso et al., 2007; Démoulin et al., 2008; Lepping et al., 2008).

Very few MCs have been observed during the present solar minimum up to now and we selected this event because it is uniquely suited to an in-depth analysis, particularly since the spacecraft are separated by a distance of order the scale size of the ejecta. The event is connected to a GOES-class B6 flare and partial halo coronal mass ejection (CME) event in active region 10956 on 20 May 2007 (Mierla et al., 2008; Kilpua et al., 2009). Thus, our event is at the lower end of the importance classification of solar eruptions as observed during solar minimum.

\section{Magnetic Cloud Event on 23 May 2007}

For data plots and a basic GS reconstruction of this event we refer the reader to Kilpua et al. (2009). The reconstructed and optimized (to be discussed later) magnetic field map from STEREO-A is shown in Figure 1(b). A 3D view of its local orientation in the heliosphere is shown in Figure 2. In this paper we do not use a genuine multi-spacecraft GS method (Sonnerup, Hasegawa, and Paschmann, 2004; Hasegawa et al., 2005, 2006; Möstl et al., 2008, 2009), i.e. we do not create combined magnetic field maps, but use GS reconstruction at one spacecraft to correlate model predictions with observations at another spacecraft (Hasegawa et al., 2004).

Because this event presents a unique opportunity to model a $\mathrm{MC}$ cross-section with two spacecraft separated by a distance of the same order as the linear scale size of the MC, we discuss four additional aspects not described in Kilpua et al. (2009): (1) The correlation between the predicted magnetic field map reconstructed from STEREO-A and the observations by Wind is quantified and discussed for all magnetic field components. (2) The Wind observations are used to optimize this correlation and clues are presented on how to handle the GS method when applied to single-spacecraft measurements. In this way an improvement on the use of GS reconstruction is suggested. (3) From the optimized magnetic field map, currents are calculated to show where the force-free condition in the MC breaks down, and (4) the number of field line turns per unit length (i.e. the twist of the field lines or equivalently how often a field line winds around the MC axis per $\mathrm{AU}$ ) is calculated for different 

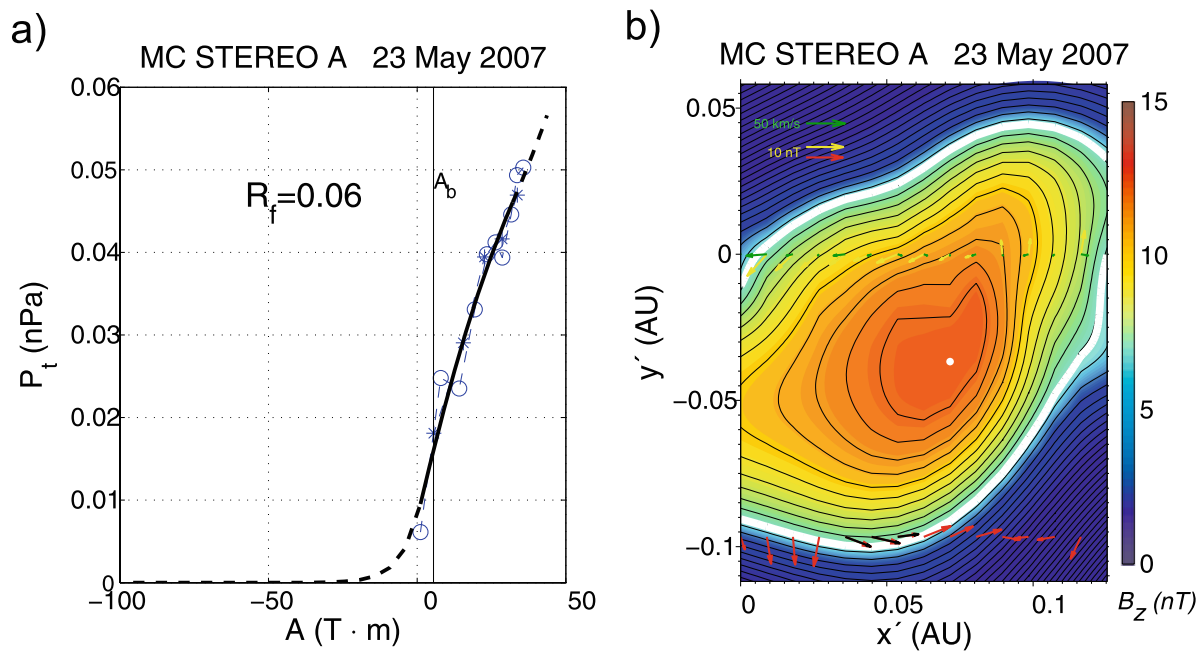

Figure 123 May $2007 \mathrm{MC}$ : (a) $P_{t}(A)$ plot with polynomial fitting function of order $f_{\mathrm{p}}=2$ (solid black) and exponential tails (dashed black). Circles denote inbound, stars outbound measurements. The vertical line $A_{b}$ delimits the interval where $P_{t}(A)$ is single-valued, corresponding to the thick white contour line in the map (right panel). (b) Reconstructed magnetic field map from STEREO-A measurements optimized using Wind observations (see text). Black contours represent transverse magnetic field lines in the paper plane, and color-coded is the $B_{z}$ component pointing out of the paper. The MC axis is at the white dot. Upper (lower) yellow (red, black) arrows are STEREO-A (Wind) observations of transverse magnetic field components, green arrows are residual velocities in the deHoffmann - Teller frame at STEREO-A. The solid white contour is the MC boundary.

distances to the axis, exhibiting an unexpected behavior, not consistent with the often used linear force-free constant- $\alpha$ Lundquist model (e.g. Goldstein, 1983; Burlaga, 1988; Lepping, Burlaga, and Jones, 1990; Leitner et al., 2007).

\subsection{Optimized Reconstruction and GS Guideline}

The GS method was originally developed for magnetopause applications (Hau and Sonnerup, 1999) and its validity has been shown by multi-spacecraft observations, e.g. flux transfer events modeled as magnetic flux ropes (Hasegawa et al., 2006) and one magnetic cloud (Liu et al., 2008). For an in-depth description of the method we refer the reader to $\mathrm{Hu}$ and Sonnerup (2002) and Sonnerup et al. (2006), but for better understanding of what follows we describe some elements necessary for the following discussion. The STEREO magnetic field data from the In situ Measurements of PArticle and CME Transients experiment (IMPACT, Luhmann et al., 2008) are rotated (implemented in SolarSoft) from the RTN coordinate system to a GSE system to facilitate the analysis using the Wind magnetic field data (also in GSE; Lepping et al., 1995). We also use plasma bulk parameters from the PLAsma and Supra-Thermal Ion Composition experiment (PLASTIC) on board STEREO (Galvin et al., 2008) and the Solar Wind Experiment (SWE) on board Wind (Ogilvie et al., 1995). The reference frame is the so-called the deHoffmann-Teller frame, moving with a velocity $\mathbf{V}_{\mathrm{HT}}$ (e.g. Khrabrov and Sonnerup (1998), see e.g. Sonnerup et al. (2006) for notes on proper use), where the flow is aligned with the magnetic field. An invariant axis $\hat{\mathbf{z}}^{\prime}$ is derived through the condition that the transverse pressure,

$$
P_{t}(A)=p+B_{z}^{\prime 2} / 2 \mu_{0},
$$


MC STEREO-A / WIND 23 May 2007

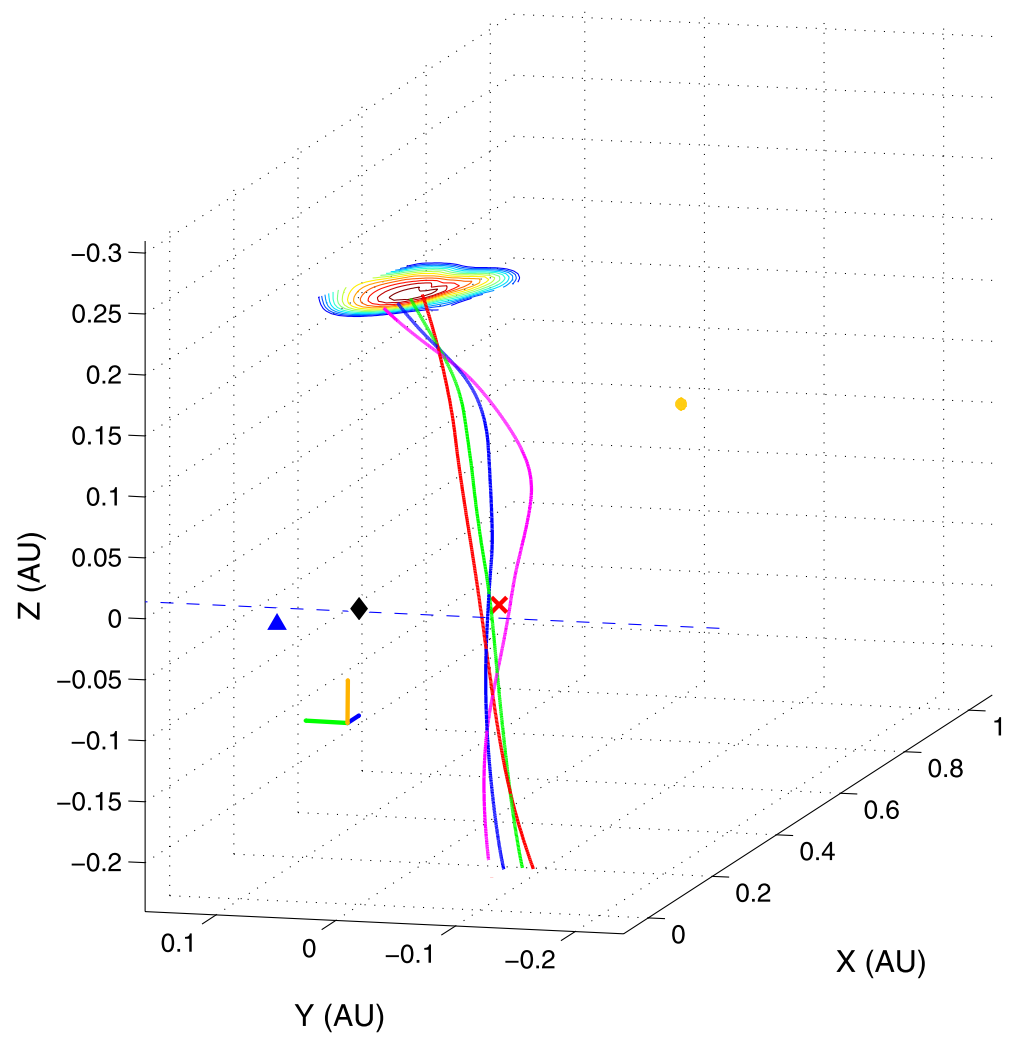

Figure 223 May 2007 MC: 3-D plot of the optimized reconstruction in the heliosphere. The positions of STEREO-A (red X), Wind (black diamond) and STEREO-B (blue triangle) are indicated. Invariance along the axis for $0.3 \mathrm{AU}$ was assumed. The coordinate system is GSE (unit vectors indicated: blue X, green Y, orange $\mathrm{Z}$ ). The Sun is the small yellow sphere (to scale).

with $p$ being the plasma pressure and $B_{z}^{\prime}$ the observed magnetic field component along $\hat{\mathbf{z}}^{\prime}$, must be as close as possible to a single-valued function (Hu and Sonnerup, 2002). A reconstruction coordinate system $\left(\hat{\mathbf{x}}^{\prime}, \hat{\mathbf{y}}^{\prime}, \hat{\mathbf{z}}^{\prime}\right)$ is established (these are the coordinates in Figure 1(b), with $\hat{\mathbf{z}}^{\prime}$ pointing out of the paper). The magnetic field observations are resampled to a number of $n_{x}$ points with a anti-aliasing low-pass filter (function "resample" in MATLAB). To numerically integrate the Grad - Shafranov equation, i.e.,

$$
\frac{\partial^{2} A}{\partial x^{\prime 2}}+\frac{\partial^{2} A}{\partial y^{\prime 2}}=-\mu_{0} \frac{\mathrm{d} P_{t}(A)}{\mathrm{d} A}=-\mu_{0} \frac{\mathrm{d}\left(p+B_{z}^{\prime 2} / 2 \mu_{0}\right)}{\mathrm{d} A}=-\mu_{0} j_{z}(A),
$$

where $A$ is the vector potential and $j_{z}(A)$ the axial current density, the right-hand side, i.e. the derivative $P_{t}(A)$, has to be known. To this end the measurements of $P_{t}(A)$ are fitted to a polynomial function, and exponential tails are used for those values of the vector potential $A$ which are not covered by observations (Figure 1(a)).

With the help of multi-spacecraft observations it is then possible to test the validity of two main method assumptions, namely, (1) time-independency, and (2) invariance along a 
particular direction. Further they show how to choose several parameters of the reconstruction which are not clear a priori and which have been thus far inferred from reconstruction of analytical data (Hu and Sonnerup, 2002). The parameters which influence the shape of the resulting magnetic field map (Figure 1(b)) are: the number of grid points $n_{x}$ along $\hat{\mathbf{x}}^{\prime}$, usually $n_{x}=15-21$ (Hu and Sonnerup, 2002; Hu et al., 2004); the order of the polynomial $f_{\mathrm{p}}$ for fitting the function $P_{t}(A)$ (often $f_{\mathrm{p}}=2-4$ ); the chosen data time interval (Hu et al., 2004), and the orientation of the invariant axis $\hat{\mathbf{z}}^{\prime}$.

An expanding $\mathrm{MC}$ would also influence the resulting magnetic field map. The expansion effect can be estimated using the expansion velocity $V_{\text {exp }}=\left(V_{l}-V_{t}\right) / 2$ with the leading edge (front boundary) velocity $V_{l}=535 \mathrm{~km} \mathrm{~s}^{-1}$ and the trailing edge (back boundary) velocity $V_{t}=453 \mathrm{~km} \mathrm{~s}^{-1}$. The deHoffmann - Teller velocity is $V_{\mathrm{HT}}=493 \mathrm{~km} \mathrm{~s}^{-1}$ and the ratio $V_{\exp } / V_{\mathrm{HT}}=0.083 \ll 1$ (Möstl et al., 2009), so the expansion effect, while clearly observed, may not be particularly significant here.

The separation vector from STEREO-A to Wind in cloud-centered coordinates is $\mathbf{s}^{\prime}=$ $[-0.0589 ;-0.0967 ;-0.0155]$ AU. Thus the probes are separated mainly along $-\hat{\mathbf{y}}^{\prime}$, making the event ideally suited to determine the cross-section of the cloud.

The Wind data have been time-shifted according to the assumption that the cloud, and thus the integration domain box, moves with constant $\mathbf{V}_{\mathrm{HT}}$ velocity (Möstl et al., 2009):

$$
t_{W}=s_{x}^{\prime} /\left(\hat{\mathbf{x}} \cdot \mathbf{V}_{\mathrm{HT}}\right),
$$

i.e. the separation vector component $s_{x}^{\prime}$ divided by the deHoffmann - Teller velocity along $\hat{\mathbf{x}}$. The result is $t_{W}=+5.38 \mathrm{~h}$. The full time interval at Wind is therefore 23 May 2007 06:20 17:48 UT; the Wind magnetic field data have been resampled also to a number of $n_{x}$ points and plotted as arrows in the $\hat{\mathbf{x}}^{\prime}-\hat{\mathbf{y}}^{\prime}$ plane in Figure 1(b) (lower trajectory). From this full interval we pick out the time range 9:36-11:12 UT corresponding to three clearly rotating arrows in Figure 1(b) between 0.036 and 0.054 AU along $\hat{\mathbf{x}}^{\prime}$ highlighted in black color (this choice will become more clear a posteriori). For every trial reconstruction a correlation coefficient is calculated between the predictions from the map (linearly interpolated between grid points) and the observed magnetic field components underlying these three arrows. Figure 3 shows this comparison (already optimized, see below), with the predicted field components at Wind position plotted as circles. From our study of this event, we draw some conclusions on how to choose the parameters quoted above correctly, as gauged by the value of $c c$ :

- $n_{x}$ : for a choice of $n_{x}=11-17$, the field map is about the same as in Figure 1(b) and $c c>0.8$. The best match was found for $n_{x}=15(c c=0.94)$.

- $f_{\mathrm{p}}$ : a polynomial of order 2 is much better than orders 3 or 4 ; for $f_{\mathrm{p}}>2$ the right hand side of Equation (2), $\mathrm{d} P_{t}(A) / \mathrm{d} A=j_{z}$, becomes larger, assuming stronger axial currents $j_{z}$ which shrinks the cross-section in the $\hat{\mathbf{y}}^{\prime}$ direction to an unreasonable small extent placing Wind completely outside the MC, contrary to what is observed (and also lowering the $c c$ ).

- Interval: the time interval (Table 1) was chosen following Hu et al. (2004), who claimed that the interval should be determined as such that $P_{t}(A)$ begins and ends at about the same $A$ value. We can completely confirm this statement, as a much longer or much shorter time interval than the one corresponding to this rule also leads to unreasonable magnetic field maps. The final STEREO-A data time interval used for the reconstruction (Figure 1(b)) is 23 May 2007 00:56-12:24 UT.

- Axis orientation $\left(\theta=58^{\circ}, \phi=220^{\circ}\right)$ : keeping the other parameters fixed and changing the orientation in only a few degrees $\left(\theta \pm 2^{\circ}\right.$, in $\left.\phi \pm 5^{\circ}\right)$ the $c c$ quickly decreases, which tells us that the invariant axis, as determined by single-spacecraft GS, is the correct one. 


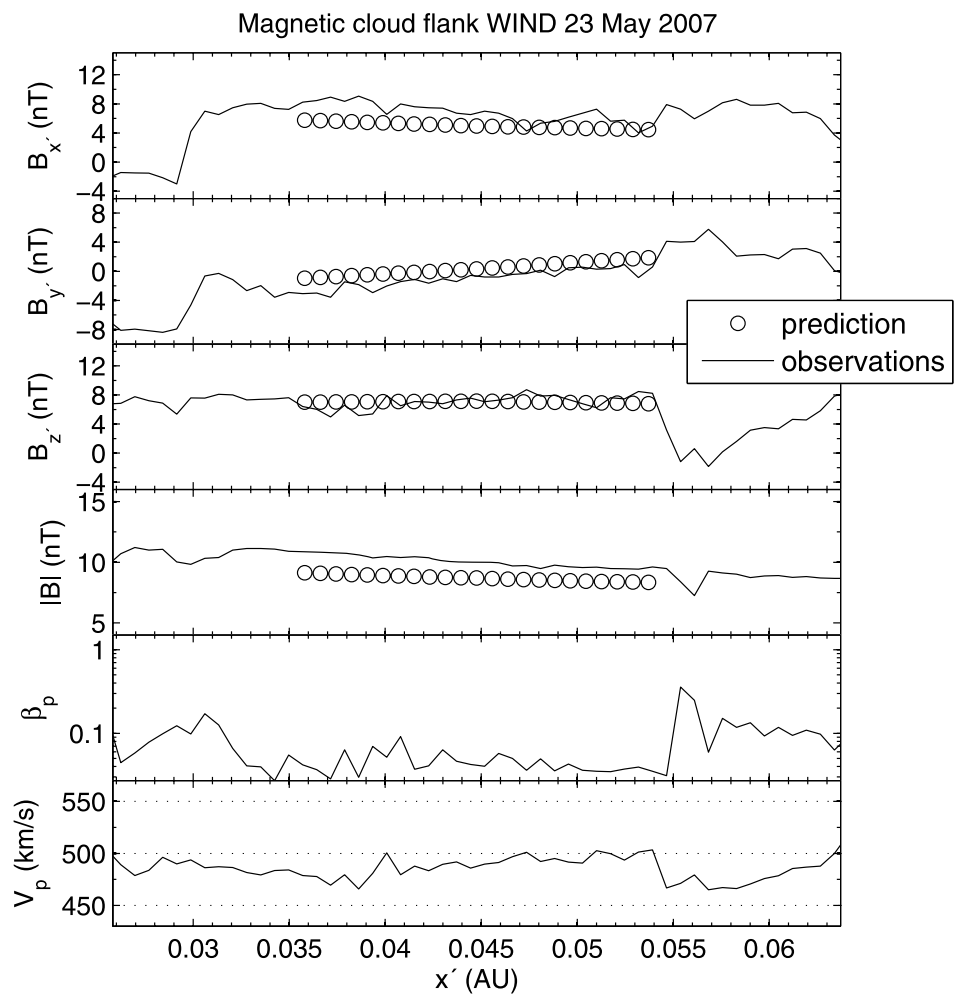

Figure 3 Comparison between the predicted magnetic field components (circles) and total field magnitude at the trajectory of Wind from the optimized STEREO-A magnetic field map (Figure 1(b)) with observations by Wind (solid lines), in reconstruction (MC) coordinates. Also shown is the proton $\beta$ (the ratio of the proton-to-magnetic pressure) and the proton bulk velocity $V_{\mathrm{p}}$ for Wind. The full $x$-axis corresponds to the Wind time interval 23 May 2007 8:40-12:08 UT. The interval for which the predictions are plotted over the observations is 23 May 2007 9:36-11:12 UT.

The time shift $t_{W}$ also depends on the orientation and only for the above given error bars there is a reasonable match between the relative timing of the predictions and observations in Figure 3.

Even though inferred from a single event, the presented results can also be considered as a guideline for the correct use of the single-spacecraft GS method for MCs. The best correlation coefficient between the filtered observations and predicted components at Wind is $c c=0.94$, for a choice of $n_{x}=15, f_{\mathrm{p}}=2$, the axis from single-spacecraft GS, and the time interval as described. One can see from Figure 3 that the decrease in $B_{x}^{\prime}$, the increase in $B_{y}^{\prime}$ and the almost constant value of $B_{z}^{\prime}$ are basically correctly modeled, although the total field strength is underestimated by $15 \%$ on average. There are also deviations between observed and predicted fields, especially in $B_{x}^{\prime}$, which stem from either the invariance or the time-independence assumption. Because the time-shift inferred from Equation (3) and the observations are consistent, we think that it is the invariance which fails and not the timeindependence. This might be attributed to the fact that the flank region of the MC is already strongly distorted by the interaction with the surrounding solar wind. 
Figure 4 The ratio of the perpendicular to the parallel currents $\varrho=\left|\boldsymbol{J}_{\perp}\right| /\left|\boldsymbol{J}_{\|}\right|$(color coded). The dotted white contour indicates the level where $\varrho=0.3$, areas with $\varrho<0.3$ are force-free by definition. Solid black contours are field lines in the $\hat{x}^{\prime}-\hat{y}^{\prime}$ plane. The solid white contour is again the MC boundary.

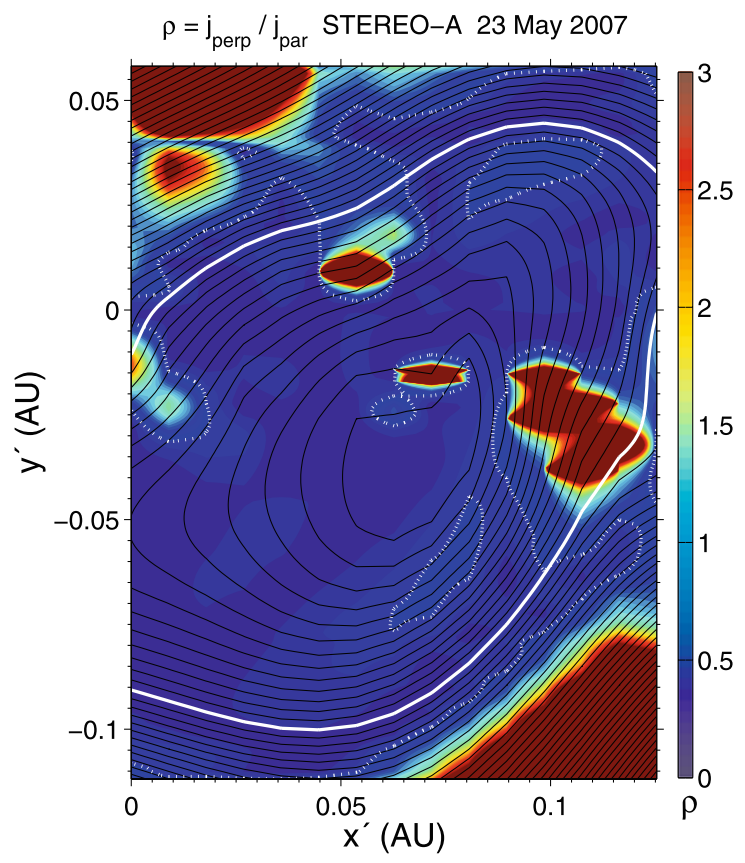

The derived axis orientation might be influenced by the minimum distance a spacecraft crosses to the MC axis (the impact parameter $p$ ). In this case $p$ equals $30 \%$ of the MC radial scale size (see Table 1). From the optimized reconstruction we infer that the derived axis orientation remains practically the same for impact parameter values up to this value of $p$ if the model assumptions are fulfilled; this has also been demonstrated for the GS method with analytic data by Hu and Sonnerup (2002), and for the minimum variance analysis method using unit vectors also with analytic data by Gulisano et al. (2007).

In summary, the separation distance between Wind and STEREO-A perpendicular to the MC axis is almost $0.1 \mathrm{AU}$, demonstrating the ability of the numerical GS solver, when used correctly, to return a reasonable magnetic field map up to this separation distance. We note that this separation is about twice that between STEREO-B and Wind for the 22 May 2007 MC event studied by Liu et al. (2008). Compared to the 22 May 2007 MC event the event we study here has the advantage that the full MC interval can be used as an input to the GS method.

\subsection{Testing the Force-Free Assumption}

From the optimized magnetic field map we calculated the currents from $\nabla \times \boldsymbol{B}=\mu_{0} \boldsymbol{J}$. The parallel current follows from $\boldsymbol{J}_{\|}=\boldsymbol{J} \cdot \boldsymbol{B} /|\boldsymbol{B}|$, and the perpendicular current is given by $\boldsymbol{J}_{\perp}=\boldsymbol{J}-\boldsymbol{J}_{\|}$. The ratio $\varrho=\left|\boldsymbol{J}_{\perp}\right| /\left|\boldsymbol{J}_{\|}\right|$is then calculated for every grid point, where we arbitrarily define a grid point to be force-free if $\varrho<0.3$, because for this ratio $\boldsymbol{J}_{\|}$still clearly dominates. Figure 4 shows the resulting map for $\varrho$. There are qualitative indications that about quarter of the cloud area is non-force-free, in particular the back part, where the MC is deformed (Figure 1(b)). Field lines which are approximately circular are force-free, as expected. We attribute this departure from the force-free state to an interaction with the trailing high-speed stream immediately following the MC interval at STEREO-A (Kilpua et al., 2009). 


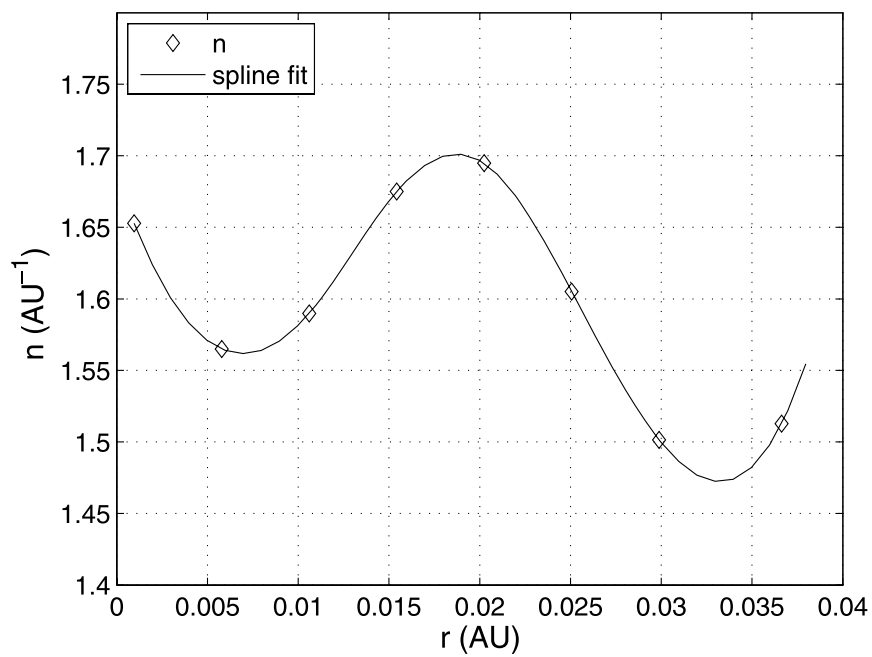

Figure 5 The number of turns $n$ a field line makes around the MC axis per AU, plotted vs. distance from the axis $r$.

Table 1 Results for the 23 May 2007 MC at STEREO-A, for optimized GS reconstruction (middle column) and force-free fitting (right column). $\theta$ is the axis inclination to the ecliptic; $\phi$ is measured from GSE-X towards GSE-Y. The scale size in the radial direction, $D$, is calculated from GS in the $\hat{x}^{\prime}-\hat{y}^{\prime}$ plane perpendicular to the axis along the spacecraft trajectory, for FF this is twice the radius $R_{0}$ obtained from the fitting procedure. $p$ is the closest distance a spacecraft passes to the MC axis (the impact parameter). For the poloidal flux $\Phi_{\mathrm{p}}$ a range of flux tube lengths, to which $\Phi_{\mathrm{p}}$ is proportional, of $L=0.5-2 \mathrm{AU}$ was taken. The total axial current $I$ is also given. The FF error bars are derived assuming a variation in $B_{0} \pm 1 \mathrm{nT}$ and in $D \pm 0.01 \mathrm{AU}$.

\begin{tabular}{lll}
\hline Parameter/method & GS & FF \\
\hline start, UT $(\Delta t)$ & $00: 56(11 \mathrm{~h} 28 \mathrm{~m})$ & $01: 02(11 \mathrm{~h} 10 \mathrm{~min})$ \\
$V_{\mathrm{HT}}, \mathrm{km} \mathrm{s}^{-1}$ & 493 & - \\
$B_{0}, \mathrm{nT}$ & 11.8 & 16.1 \\
$\theta, \mathrm{deg}$ & -58 & -68 \\
$\phi, \mathrm{deg}$ & 220 & 281 \\
$H$ & $\mathrm{R}$ & $\mathrm{R}$ \\
$D, \mathrm{AU}$ & 0.125 & 0.103 \\
$p, \mathrm{AU}$ & 0.037 & 0.023 \\
$\Phi_{t}, 10^{21} \mathrm{Mx}$ & 0.30 & $0.13 \pm 0.06$ \\
$\Phi_{\mathrm{p}}, 10^{21} \mathrm{Mx}$ & $0.28-1.11$ & $0.33-1.8$ \\
$I, \mathrm{MA}$ & 323 & $322 \pm 20$ \\
\hline
\end{tabular}

\subsection{Field Line Turns}

The number of turns $n$ per AU for various distances from the MC axis $r$ was calculated from the optimized map and the result is shown in Figure 5. For this we assumed invariance and followed magnetic field lines around the MC axis until they closed on themselves (similar to 
Figure 2) to determine the so-called pitch. This is the axial length of a field line in AU that encircles the axis once, and $n$ is the inverse of the pitch. For the linear force-free, constant- $\alpha$ Lundquist model, $n$ is a monotonically increasing function of radial distance from the axis. For the MC under discussion, the behavior is not monotonic: a steady increase is only found between $r=0.005-0.02$ AU (Figure 5), but then $n$ declines. This means that the outer field lines are less twisted than the inner ones, which is at variance with the linear force-free model. Another event with a decrease in $n$ has been reported by Hu and Sonnerup (2002). However, this is the first time that a magnetic cloud has been reconstructed which shows both first increasing and then decreasing $n$, and the optimization procedure gives us confidence in these results. Field lines for which $r>0.037 \mathrm{AU}$ do not close on themselves in the magnetic field map so we cannot determine $n$. The number of turns $n=1.5-1.7 \mathrm{AU}^{-1}$ does not vary much, for a length $L=2.5 \mathrm{AU}$ the full number of turns is about four. An example of a small-scale flux rope with field line twist independent of $r$ (a so-called "Gold-Hoyle" tube) was studied by Farrugia et al. (1999), and another MC event (18 October 1995) with almost constant $n$ was discussed by $\mathrm{Hu}$ and Sonnerup (2002).

\section{Summary and Conclusions}

In this paper we demonstrated several issues related to the method of Grad-Shafranov reconstruction and its application to the 23 May 2007 magnetic cloud event, building on the work of Kilpua et al. (2009). We obtain the following new results. We used a novel technique for the first time in the interplanetary context in which a reconstructed magnetic field map is optimized through correlation techniques, and demonstrated the ability of the numerical solver used to return reliable results up to a spacecraft separation distance of $\approx 0.1 \mathrm{AU}$, which in this case corresponds to $80 \%$ of the clouds scale size in the radial direction. From this we answered some open questions on the correct use of the method and presented guidelines for its future use in magnetic clouds. From the optimized field map, currents were derived to show where in the magnetic cloud the force-free assumption breaks down. How the field lines are twisted in the non-force-free model was also discussed, exhibiting in this case an interesting behavior which is inconsistent with the popular force-free Lundquist model.

For comparison, the results of the optimized GS reconstruction and force-free fitting (FF, Lepping, Burlaga, and Jones, 1990) for global MC parameters are shown in Table 1. The good quality of the force-free fit is demonstrated in Figure 6. There is in general good agreement between the two methods, but there is a difference in the angle $\phi$ of 60 degrees (which could arise from the high inclination), the axial flux is underestimated by FF by about a factor of 2 and the axial field $B_{0}$ is also clearly higher for FF. But on the inclination, impact parameter, poloidal flux and axial current the agreement is very good. This is consistent with the work of Dasso et al. (2003) who found that various cylindrical models used for fitting the magnetic field profile of MCs also lead to similar magnetic fluxes. It could be that a systematic difference which arises from the deformed cross-section, the different twist of the field lines and the non-force-free treatment by GS affects some parameters more than others. We also note that the method without optimization, i.e. a "blind" reconstruction, gives quite similar results (see also Figure 6c in Kilpua et al., 2009).

However, we think the confidence in the determination of the main cloud parameters, especially in the orientation, clearly increases with multi-spacecraft observations because for a good consistency between model predictions and observations there is not much variation possible in global parameters. The magnetic field map shows the main part of the cloud 
Stereo A 2007 DOY 142-143

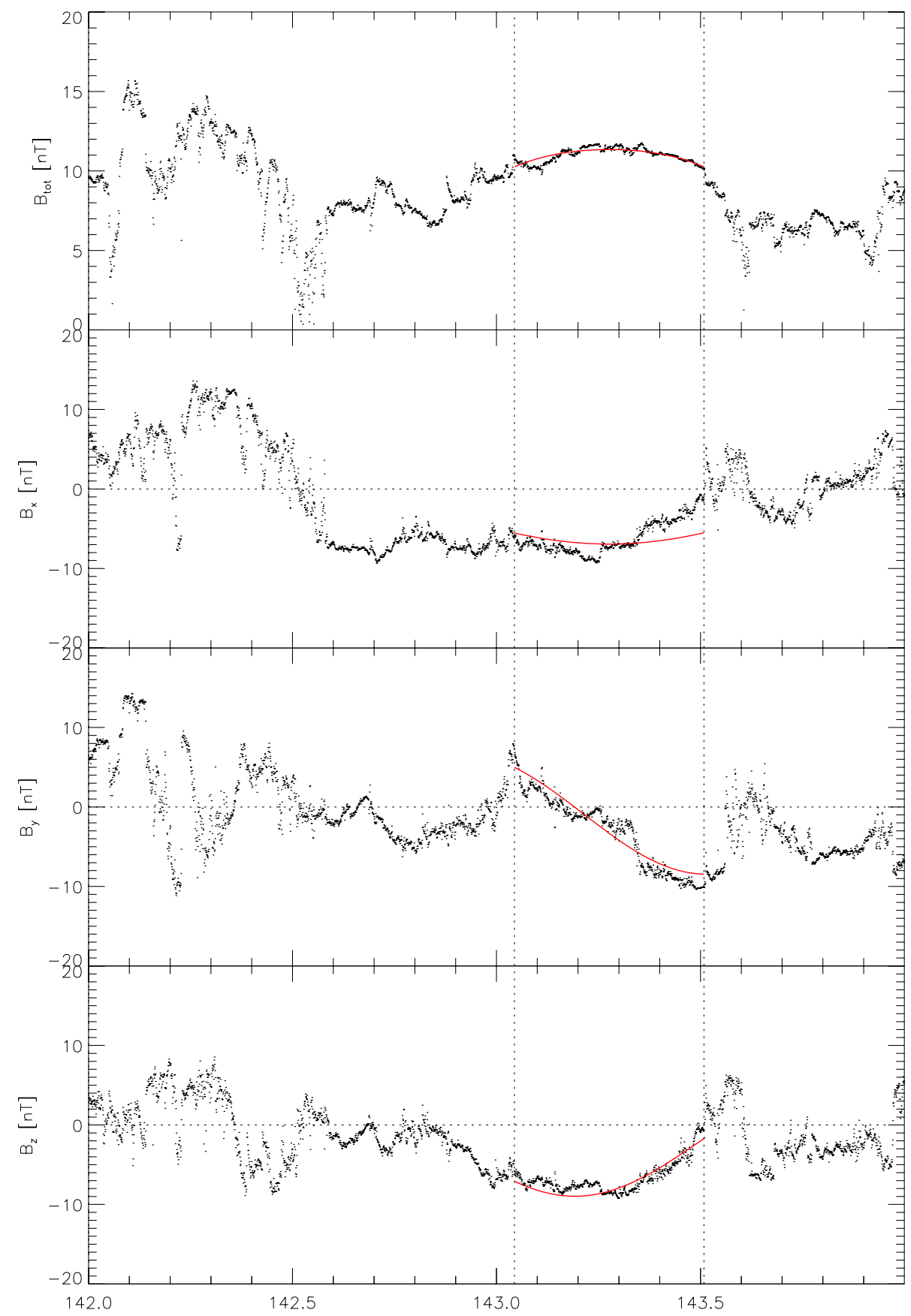

Figure 6 STEREO-A magnetic field components in GSE coordinates fitted by the linear force-free model (red solid line). 
which seems to extend more in the $-\hat{\mathbf{x}}^{\prime}$ and $+\hat{\mathbf{x}}^{\prime}$ directions outside of the map (Figure 1(b)), and thus the fluxes and the total axial current in Table 1 are still underestimated. However, we think that the main spatial extent of the cloud is well covered and the results are quite close to reality (for further discussions see also Möstl et al., 2009).

The often criticized circular, linear Lundquist model seems nevertheless to be a good description of the core region of at least some MCs, for which evidence has also been found by e.g. Dasso et al. (2005). The present method still assumes an invariant axis and time-independence. With multi-spacecraft observations, such as presented here, correlation techniques can be used to test these assumptions. These correlations showed that timeindependence is quite valid for a time-lapse of about 5 hours from STEREO-A to Wind, and that there were some important departures from the assumption of invariance. This study was a first step to show that the method is indeed capable of returning an approximate shape of a magnetic cloud cross-section. In the future, it would be necessary to develop a complete 3D torus-shaped model of magnetic clouds which can then be constrained by multi-spacecraft observations of several events. In summary, the analysis shows the usefulness of widely separated multi-spacecraft observations in determining the three-dimensional shape of magnetic clouds (see also the suggestions by Marubashi and Lepping, 2007). Here, we found evidence that a magnetic cloud cross-section has the shape of a helical magnetic flux rope consisting of "distorted ellipses".

The optimized reconstruction method has given us a picture of the magnetic cloud 3Dconfiguration near Earth (Figure 2). The corresponding CME event on 20 May 2007 has been discussed by Mierla et al. (2008) using data from Inner Coronagraph (COR1) of the Sun Earth Connection Coronal and Heliospheric Investigation (SECCHI) experiment from two view-points, inferring the CME direction and de-projected velocity. Kilpua et al. (2009) showed that this de-projected velocity matches much better the transit time of the MC than the projected velocity. Additionally, Mierla et al. (2008) were able to estimate the CME propagation direction as 2 degrees east of the Sun-Earth line between 2.4 to $6 R_{\odot}$, and the latitude as -30 degree to the ecliptic. At Earth, the MC axis passes west of Earth by approximately 3 degrees (see Figure 2, and Figures 1 and 7 in Kilpua et al., 2009) but seems to have its apex indeed below the ecliptic. Thus the longitude estimation was correct to within 5 degrees, indicating that the MC was not deflected much from the radial direction between $6 R_{\odot}$ and $1 \mathrm{AU}$. However, this difference might already be decisive in forecasting the geo-effects of a highly inclined magnetic cloud from observations near the Sun.

Acknowledgements C.M. and M.L. acknowledge the Austrian Fonds zur Förderung der wissenschaftlichen Forschung under projects P20145-N16 and P20131-N16 for funding. C.M. also thanks the Steiermärkische Sparkasse, the University of Graz and the government of Styria for support. This work is supported by NASA contracts NAS5-00132 to the University of New Hampshire for the STEREO PLASTIC investigation and NAS5-03131 to the University of California, Berkeley, for the STEREO IMPACT investigation. It is also supported by NASA Wind grants to UNH NNG06GD41G and NNX08AD11G. We also acknowledge the use of Wind data provided by the magnetometer and the solar wind experiment teams at GSFC. Additionally, we would like to thank the referee and the guest editors for their very thorough reading of the manuscript.

Open Access This article is distributed under the terms of the Creative Commons Attribution Noncommercial License which permits any noncommercial use, distribution, and reproduction in any medium, provided the original author(s) and source are credited.

\section{References}

Bothmer, V., Schwenn, R.: 1998, The structure and origin of magnetic clouds in the solar wind. Ann. Geophys. 16, 1 . 
Burlaga, L.F.: 1988, Magnetic clouds and force-free fields with constant alpha. J. Geophys. Res. 93, 7217.

Burlaga, L.F., Lepping, R.P., Jones, J.A.: 1990, Global configuration of a magnetic cloud. In: Russell, C.T., Priest, E.R., Lee, L.C. (eds.) Physics of Magnetic Flux Ropes, Geophysical Monograph Ser. 58, AGU, Washington DC, 377.

Burlaga, L., Sittler, E., Mariani, F., Schwenn, R.: 1981, Magnetic loop behind an interplanetary shock Voyager, Helios, and IMP 8 observations. J. Geophys. Res. 86, 6673.

Cane, H.V., Richardson, I.G.: 2003, Interplanetary coronal mass ejections in the near-Earth solar wind during 1996-2002. J. Geophys. Res. (Space Phys.) 108, 1156. doi:10.1029/2002JA009817.

Dasso, S., Mandrini, C.H., Démoulin, P., Farrugia, C.J.: 2003, Magnetic helicity analysis of an interplanetary twisted flux tube. J. Geophys. Res. (Space Phys.) 108, 1362. doi:10.1029/2003JA009942.

Dasso, S., Gulisano, A.M., Mandrini, C.H., Démoulin, P.: 2005, Model-independent large-scale magnetohydrodynamic quantities in magnetic clouds. Adv. Space Res. 35, 2172. doi:10.1016/j.asr.2005.03.054.

Dasso, S., Mandrini, C.H., Démoulin, P., Luoni, M.L.: 2006, A new model-independent method to compute magnetic helicity in magnetic clouds. Astron. Astrophys. 455, 349. doi:10.1051/0004-6361:20064806.

Dasso, S., Nakwacki, M.S., Démoulin, P., Mandrini, C.H.: 2007, Progressive transformation of a flux rope to an ICME. Comparative analysis using the direct and fitted expansion methods. Solar Phys. 244, 115. doi:10.1007/s11207-007-9034-2.

Démoulin, P.: 2008, A review of the quantitative links between CMEs and magnetic clouds. Ann. Geophys. 26, 3113 .

Démoulin, P., Nakwacki, M.S., Dasso, S., Mandrini, C.H.: 2008, Expected in situ velocities from a hierarchical model for expanding interplanetary coronal mass ejections. Solar Phys. 250, 347. doi:10.1007/s11207-008-9221-9.

Farrugia, C.J.: 1997, Recent work on modelling the global field line topology of interplanetary magnetic clouds: A review. In: Crooker, N., Joselyn, J.A., Feynmann, J. (eds.) Coronal Mass Ejections, Geophysical Monograph Ser. 99, AGU, Washington DC, 177.

Farrugia, C.J., Osherovich, V.A., Burlaga, L.F.: 1995, Magnetic flux rope versus the spheromak as models for interplanetary magnetic clouds. J. Geophys. Res. 100, 12293.

Farrugia, C.J., Burlaga, L.F., Osherovich, V.A., Lepping, R.P.: 1992, A comparative study of dynamically expanding force-free, constant-alpha magnetic configurations with applications to magnetic clouds. In: Marsch, E., Schwenn, R. (eds.) Solar Wind Seven, Proc. 3rd, COSPAR Colloq., 611.

Farrugia, C.J., Burlaga, L.F., Osherovich, V.A., Richardson, I.G., Freeman, M.P., Lepping, R.P., Lazarus, A.J.: 1993a, A study of an expanding interplanetary magnetic cloud and its interaction with the Earth's magnetosphere - The interplanetary aspect. J. Geophys. Res. 98, 7621.

Farrugia, C.J., Richardson, I.G., Burlaga, L.F., Lepping, R.P., Osherovich, V.A.: 1993b, Simultaneous observations of solar MeV particles in a magnetic cloud and in the Earth's northern tail lobe - Implications for the global field line topology of magnetic clouds and for the entry of solar particles into the magnetosphere during cloud passage. J. Geophys. Res. 98, 15497.

Farrugia, C.J., Janoo, L.A., Torbert, R.B., Quinn, J.M., Ogilvie, K.W., Lepping, R.P., Fitzenreiter, R.J., Steinberg, J.T., Lazarus, A.J., Lin, R.P., Larson, D., Dasso, S., Gratton, F.T., Lin, Y., Berdichevsky, D.: 1999, A uniform-twist magnetic flux rope in the solar wind. In: Suess, S.T., Gary, G.A., Nerney, S.F. (eds.) Solar Wind Nine, AIP Conf. Ser. 471, 745.

Farrugia, C.J., Vasquez, B., Richardson, I.G., Torbert, R.B., Burlaga, L.F., Biernat, H.K., Mühlbachler, S., Ogilvie, K.W., Lepping, R.P., Scudder, J.D., et al.: 2001, A reconnection layer associated with a magnetic cloud. Adv. Space Res. 28, 759.

Galvin, A.B., Kistler, L.M., Popecki, M.A., Farrugia, C.J., Simunac, K.D.C., Ellis, L., Möbius, E., Lee, M.A., Boehm, M., Carroll, J., et al.: 2008, The plasma and suprathermal ion composition (PLASTIC) investigation on the STEREO observatories. Space Sci. Rev., 136, 437.

Goldstein, H.: 1983, On the field configuration in magnetic clouds. In: Neugebauer, M. (ed.) Solar Wind Five, NASA Conf. Publ. CP-2280, 731.

Gopalswamy, N.: 2006, Properties of interplanetary coronal mass ejections. Space Sci. Rev. 124, 145. doi:10.1007/s11214-006-9102-1.

Gosling, J.T., Eriksson, S., McComas, D.J., Phan, T.D., Skoug, R.M.: 2007, Multiple magnetic reconnection sites associated with a coronal mass ejection in the solar wind. J. Geophys. Res. (Space Phys.) 112, A08106. doi:10.1029/2007JA012418.

Gulisano, A.M., Dasso, S., Mandrini, C.H., Démoulin, P.: 2007, Estimation of the bias of the minimum variance technique in the determination of magnetic clouds global quantities and orientation. Adv. Space Res. 40, 1881. doi:10.1016/j.asr.2007.09.001.

Hasegawa, H., Sonnerup, B., Dunlop, M., Balogh, A., Haaland, S., Klecker, B., Paschmann, G., Lavraud, B., Dandouras, I., Rème, H.: 2004, Reconstruction of two-dimensional magnetopause structures from Cluster observations: Verification of method. Ann. Geophys. 22, 1251. 
Hasegawa, H., Sonnerup, B.U.Ö., Klecker, B., Paschmann, G., Dunlop, M.W., Rème, H.: 2005, Optimal reconstruction of magnetopause structures from Cluster data. Ann. Geophys. 23, 973.

Hasegawa, H., Sonnerup, B.U.Ö., Owen, C.J., Klecker, B., Paschmann, G., Balogh, A., Rème, H.: 2006, The structure of flux transfer events recovered from Cluster data. Ann. Geophys. 24, 603.

Hau, L.N., Sonnerup, B.U.Ö.: 1999, Two-dimensional coherent structures in the magnetopause: Recovery of static equilibria from single-spacecraft data. J. Geophys. Res. 104, 6899. doi:10.1029/1999JA900002.

Hu, Q., Sonnerup, B.U.Ö.: 2002, Reconstruction of magnetic clouds in the solar wind: Orientations and configurations. J. Geophys. Res. (Space Phys.) 107, 1142. doi:10.1029/2001JA000293.

Hu, Q., Smith, C.W., Ness, N.F., Skoug, R.M.: 2004, Multiple flux rope magnetic ejecta in the solar wind. J. Geophys. Res. (Space Phys.) 109, A03102. doi:10.1029/2003JA010101.

Jian, L., Russell, C.T., Luhmann, J.G., Skoug, R.M.: 2006, Properties of interplanetary coronal mass ejections at one AU during 1995 - 2004. Solar Phys. 239, 393. doi:10.1007/s11207-006-0133-2.

Kaiser, M.L., Kucera, T.A., Davila, J.M., St. Cyr, O.C., Guhathakurta, M., Christian, E.: 2008, The STEREO mission: An introduction. Space Sci. Rev. 136, 5. doi:10.1007/s11214-007-9277-0.

Khrabrov, A.V., Sonnerup, B.U.Ö.: 1998, deHoffmann - Teller analysis. In: Paschmann, G., Daly, P.W. (eds.) Analysis Methods for Multi-Spacecraft Data, ISSI Sience Rep. Ser. 1, ESA, Noordwijk 221.

Kilpua, E.K.J., Liewer, P., Farrugia, C., Luhmann, J., Möstl, C., Li, Y., Liu, Y., Lynch, B., Vourlidas, A., Acuna, M., Galvin, A.B., Larson, D., Russell, C., Sauvaud, J.: 2009, Multispacecraft observations of magnetic clouds and their solar origins May 19-23, 2007. Solar Phys. 254, 325. doi:10.1007/s11207-008-9300-y.

Leitner, M., Farrugia, C.J., Möstl, C., Ogilvie, K.W., Galvin, A.B., Schwenn, R., Biernat, H.K.: 2007, Consequences of the force-free model of magnetic clouds for their heliospheric evolution. J. Geophys. Res. (Space Phys.) 112, A06113. doi:10.1029/2006JA011940.

Lepping, R.P., Burlaga, L.F., Jones, J.A.: 1990, Magnetic field structure of interplanetary magnetic clouds at 1 AU. J. Geophys. Res. 95, 11957.

Lepping, R.P., et al.: 1995, The wind magnetic field investigation, Space Sci. Rev., 71, 207.

Lepping, R.P., Wu, C.C., Berdichevsky, D.B., Ferguson, T.: 2008, Estimates of magnetic cloud expansion at 1 AU. Ann. Geophys. 26, 1919.

Liu, Y., Richardson, J.D., Belcher, J.W., Wang, C., Hu, Q., Kasper, J.C.: 2006, Constraints on the global structure of magnetic clouds: Transverse size and curvature. J. Geophys. Res. (Space Phys.) 111, A12S03. doi:10.1029/2006JA011890.

Liu, Y., Luhmann, J.G., Huttunen, K.E.J., Lin, R.P., Bale, S.D., Russell, C.T., Galvin, A.B.: 2008, Reconstruction of the 2007 May 22 magnetic cloud: How much can we trust the flux-rope geometry of CMEs? Astrophys. J. Lett. 677, L133. doi:10.1086/587839.

Longcope, D., Beveridge, C., Qiu, J., Ravindra, B., Barnes, G., Dasso, S.: 2007, Modeling and measuring the flux reconnected and ejected by the two-ribbon flare/CME event on 7 November 2004. Solar Phys. 244, 45. doi:10.1007/s11207-007-0330-7.

Luhmann, J. G., et al.: 2008, STEREO IMPACT investigation goals, measurements, and data products overview. Space Sci. Rev., 136, 117.

Marubashi, K.: 1997, Interplanetary flux ropes and solar filaments. In: Crooker, N., Joselyn, J.A., Feynman, J. (eds.) Coronal Mass Ejections, Geophysical Monograph Ser. 99, AGU, Washigton DC, 147.

Marubashi, K., Lepping, R.P.: 2007, Long-duration magnetic clouds: a comparison of analyses using torusand cylinder-shaped flux rope models. Ann. Geophys. 25, 2453.

Mierla, M., Davila, J., Thompson, W., Inhester, B., Srivastava, N., Kramar, M., St. Cyr, O.C., Stenborg, G., Howard, R.A.: 2008, A quick method for estimating the propagation direction of coronal mass ejections using STEREO-COR1 images. Solar Phys. 252, 385. doi:10.1007/s11207-008-9267-8.

Möstl, C., Miklenic, C., Farrugia, C.J., Temmer, M., Veronig, A., Galvin, A.B., Vršnak, B., Biernat, H.K.: 2008, Two-spacecraft reconstruction of a magnetic cloud and comparison to its solar source. Ann. Geophys. 26, 3139.

Möstl, C., Farrugia, C.J., Miklenic, C., Temmer, M., Galvin, A.B., Luhmann, J.G., Huttunen, K.E.J., Leitner, M., Nieves-Chinchilla, T., Veronig, A., Biernat, H.K.: 2009, Multi-spacecraft recovery of a magnetic cloud and its origin from magnetic reconnection on the Sun. J. Geophys. Res. (Space Phys.) 114, A04102. doi:10.1029/2008JA013657.

Mulligan, T., Russell, C.T.: 2001, Multispacecraft modeling of the flux rope structure of interplanetary coronal mass ejections: Cylindrically symmetric versus nonsymmetric topologies. J. Geophys. Res. 106, 10581. doi:10.1029/2000JA900170.

Mulligan, T., Russell, C.T., Anderson, B.J., Lohr, D.A., Rust, D., Toth, B.A., Zanetti, L.J., Acuna, M.H., Lepping, R.P., Gosling, J.T.: 1999, Intercomparison of NEAR and Wind interplanetary coronal mass ejection observations. J. Geophys. Res. 104, 28217.

Ogilvie, K.W., Chornay, D.J., Fritzenreiter, R.J., Hunsaker, F., Keller, J., Lobell, J., Miller, G., Scudder, J.D., Sittler, E.C. Jr., Torbert, R.B., et al.: 1995, SWE, A comprehensive plasma instrument for the wind spacecraft. Space Sci. Rev., 71, 55. 
Qiu, J., Hu, Q., Howard, T.A., Yurchyshyn, V.B.: 2007, On the magnetic flux budget in low-corona magnetic reconnection and interplanetary coronal mass ejections. Astrophys. J. 659, 758. doi:10.1086/512060.

Reinard, A.A.: 2008, Analysis of interplanetary coronal mass ejection parameters as a function of energetics, source location, and magnetic structure. Astrophys. J. 682, 1289. doi:10.1086/589322.

Riley, P., Crooker, N.U.: 2004, Kinematic treatment of coronal mass ejection evolution in the solar wind. Astrophys. J. 600, 1035. doi:10.1086/379974.

Riley, P., Linker, J.A., Lionello, R., Mikić, Z., Odstrcil, D., Hidalgo, M.A., Cid, C., Hu, Q., Lepping, R.P., Lynch, B.J., Rees, A.: 2004, Fitting flux ropes to a global MHD solution: A comparison of techniques. J. Atmos. Solar-Terr. Phys. 66, 1321. doi:10.1016/j.jastp.2004.03.019.

Riley, P., Schatzman, C., Cane, H.V., Richardson, I.G., Gopalswamy, N.: 2006, On the rates of coronal mass ejections: Remote solar and in situ observations. Astrophys. J. 647, 648. doi:10.1086/505383.

Shodhan, S., Crooker, N.U., Kahler, S.W., Fitzenreiter, R.J., Larson, D.E., Lepping, R.P., Siscoe, G.L., Gosling, J.T.: 2000, Counterstreaming electrons in magnetic clouds. J. Geophys. Res. 105, 27261. doi:10.1029/2000JA000060.

Sonnerup, B.U.Ö., Hasegawa, H., Paschmann, G.: 2004, Anatomy of a flux transfer event seen by cluster. Geophys. Res. Lett. 31, L11803. doi:10.1029/2004GL020134.

Sonnerup, B.U.Ö., Hasegawa, H., Teh, W.L., Hau, L.N.: 2006, Grad-Shafranov reconstruction: An overview. J. Geophys. Res. (Space Phys.) 111, A09204. doi:10.1029/2006JA011717. 